\title{
Bewertung des Schwingungsdosiswertes und Fahrkomfort im PKW unter Unsicherheiten
}

\section{Evaluation of the Vibration Dose Value (VDV) and riding comfort in a passenger car under random excitations}

\author{
M.Sc. K. Zerle, Dr.-Ing. K. Sepahvand, Prof. Dr.-Ing. S. Marburg, \\ Lehrstuhl für Akustik mobiler Systeme, TU München
}

\section{Kurzfassung}

Diese Arbeit befasst sich mit der Bewertung der Vibrationsbelastung, der ein Insasse in einem Auto während der Fahrt ausgesetzt ist. Es wird insbesondere der Schwingungsdosiswert (VDV) betrachtet. Zur Analyse wird ein Mehrkörpersimulationsmodell eines Personenkraftwagens entwickelt. Das Hauptziel der Arbeit ist die Anwendung von Methoden zur stochastischen Simulation, denn die Schwingungsanregung an den Rädern wird als zufällig angenommen. Da die konventionelle, stichprobenbasierte Monte Carlo Methode nicht akzeptabel hohe Rechenzeiten verursacht, wird Polynomial Chaos Entwicklung mit der Kollokationsmethode angewandt. Es werden Simulationsergebnisse generiert, mit denen der Schwingungsdosiswert bewertet werden kann. Die Simulationsergebnisse von Monte Carlo Methode und Kollokationsmethode werden verglichen. Hintergrundinformationen und Zusammenhänge sowohl zum Thema Schwingungskomfort und Auswirkungen von Vibrationen auf den menschlichen Körper, als auch die Grundlagen stochastischer Simulation, werden erläutert.

Die Relevanz und die Mächtigkeit der Anwendung stochastischer Simulationsmethoden für die Berechnung des Schwingungsdosiswerts bei zufälliger Schwingungsanregung wird aufgezeigt. Des Weiteren wird die Genauigkeit der Ergebnisse der Kollokationsmethode bestätigt. Hinsichtlich der benötigten Rechenzeiten kann der Kollokationsmethode ein großer Vorteil gegenüber der Monte Carlo Methode attestiert werden.

\section{Abstract}

This study deals with the evaluation of the vibration the passenger of a car is exposed to while riding. In particular, the Vibration Dose Value (VDV) is considered. A multibody simulation model of a passenger car is developed. As uncertainty within the excitation at the car's wheels shall be respected, the main goal of this work is the application of stochastic simulation methods. As the conventional sampling-based Monte Carlo Method generates an 
unacceptably high computational cost, generalized Polynomial Chaos using the non-sampling Collocation Method is applied to obtain results, with which the Vibration Dose Value can be evaluated. The simulation results of Monte Carlo Method and Collocation Method are compared. Information is given on comfort analysis and human vibration exposure, and relations concerning stochastic simulation are explained.

The study confirms the importance and mightiness of stochastic simulation for the calculation of the Vibration Dose Value when random excitations are present. Furthermore, the accuracy of the results obtained by applying generalized Polynomial Chaos expansion is approved. Concerning computational cost, the outcomes of this work attest the great advantage of the Collocation Method over the conventional sampling-based Monte Carlo Method.

\section{Einleitung}

Der Mensch ist täglich Schwingungen ausgesetzt. Beispielsweise findet die Exposition beim Reisen auf der Straße statt. Zur Beurteilung der Schwingungseinwirkungen auf den Körper des Menschen kann der Schwingungsdosiswert (Vibration Dose Value - VDV) betrachtet werden. Die Schwingungsanregung ist oft stochastisch, und die Übertragung von Schwingungen auf den menschlichen Körper sowie deren Wirkung sind von der Körperhaltung und individuellen Merkmalen wie Körperbau, Alter, Geschlecht oder Gesundheitszustand abhängig. Dieser Artikel befasst sich mit der Bewertung der Vibrationsbelastung unter Berücksichtigung von Unsicherheiten, der ein Insasse in einem Auto bei der Fahrt auf unebenem Untergrund ausgesetzt ist.

Während die Belastung durch das Arbeiten mit Werkzeugen zumeist als Hand-ArmSchwingungen klassifiziert werden kann, handelt es sich bei der Fahrt im PKW vor allem um Ganzkörper-Schwingungen. Es sind demnach nicht nur einzelne Körperteile, welche in unmittelbarem Kontakt mit einer vibrierenden Oberfläche stehen, Vibrationen ausgesetzt, sondern es ist vielmehr der gesamte Körper betroffen. Die Folgen von GanzkörperSchwingungen reichen von Komforteinbußen über Einschränkungen hinsichtlich der Ausübung anspruchsvoller Tätigkeiten bis hin zu gesundheitlichen Risiken. Häufige Folgeerscheinungen von exzessiver Ganzkörper-Vibrationsbelastung in Fahrzeugen sind Rückenschmerzen im Bereich der Lendenwirbelsäule und Erkrankungen des Bewegungsapparates im Allgemeinen. Die Folgen von Ganzkörper-Schwingungen hängen von verschiedenen Faktoren ab, wie der Schwingungsamplitude, der Frequenz, sowie der Dauer der Vibrationsbelastung.

Zur Bewertung der Ganzköper-Vibrationsbelastung in Fahrzeugen wird häufig der Schwingungsdosiswert VDV (Vibration Dose Value) herangezogen [1] 


$$
V D V=\sqrt[4]{\int_{0}^{T} a_{w}^{4}(t) \mathrm{d} t}
$$

mit der Messdauer $T$ und der frequenzgewichteten Beschleunigung $a_{w}(t)$ zur Zeit $t$. Der Schwingungsdosiswert ist ein kumulativer Messwert mit der Einheit $m / s^{1.75}$. Diese Messgröße wird in der vorliegenden Arbeit zur Bewertung der Schwingungsbelastung der Insassen eines PKW genutzt.

Zur Beachtung, Handhabung und Klassifizierung von Ganzkörper-Schwingungen sind, neben unzähligen wissenschaftlichen Veröffentlichungen, verschiedene nationale und internationale Normen verfügbar, beispielsweise ISO 2631, BS 6841 und VDI 2057. In diesen Richtlinien werden unter anderem Grenzen für die menschliche Schwingungsaussetzung vorgeschlagen, um negative Auswirkungen zu verhindern.

Zahlreiche wissenschaftliche Arbeiten nutzen experimentelle Ansätze oder deterministische Simulationsmethoden, um die menschliche Ganzkörper-Vibrationsbelastung im PKW zu bewerten. Nur in sehr wenigen Veröffentlichungen wird stochastische Simulation angewandt, um beispielsweise Unsicherheiten im Simulationsmodell oder der Oberfläche der Fahrbahn zu berücksichtigen [2, 3]. Jedoch wurde bislang kein Ansatz zur nicht sampling-basierten Berechnung des Schwingungsdosiswertes (Vibration Dose Value - VDV) bei der Fahrt im PKW unter Unsicherheiten verfolgt, sondern es wurde stets die sampling-basierte Monte Carlo Simulationsmethode angewandt. Dies ist unter anderem durch den nach wie vor geringen Bekanntheitsgrad sogenannter „non-sampling Methoden“, sowie durch die damit verbundene anspruchsvollere Mathematik begründet.

Die Anwendung der Monte Carlo Methode für stochastische Simulation geht einher mit einem sehr hohen Rechenaufwand, da dieser Ansatz sehr ineffizient ist. Deshalb kann diese sampling-basierte Methode unter Anwendung detaillierter Simulationsmodelle in der Praxis kaum eingesetzt werden, denn die Rechenzeiten würden inakzeptabel hoch.

In dieser Arbeit soll daher die nicht sampling-basierte Polynomial Chaos Methode angewandt werden, um die Schwingungsbelastung der Insassen eines PKW während der Fahrt zu bewerten. Insbesondere wird die Kollokationsmethode eingesetzt, um die Unsicherheiten in der Anregung durch das zufällige Oberflächenprofil der Fahrbahn zu berücksichtigen.

Dieser Artikel ist wie folgt aufgebaut. Im folgenden Abschnitt wird kurz auf Methoden zur stochastischen Simulation eingegangen. Anschließend wird unter Punkt 3 das verwendete Mehrkörper-Simulationsmodell des PKW vorgestellt. Ergebnisse der numerischen Simulation werden schließlich in Abschnitt 4 gezeigt, bevor unter Punkt 5 eine Zusammenfassung erfolgt. 


\section{Stochastische Simulation}

Ergebnisse, welche mit deterministischer Simulation gewonnen werden, haben, verglichen mit stochastischer Simulation, nur eine sehr begrenzte Aussagekraft. Dies geht unmittelbar aus der Grafik in Bild 1 hervor.
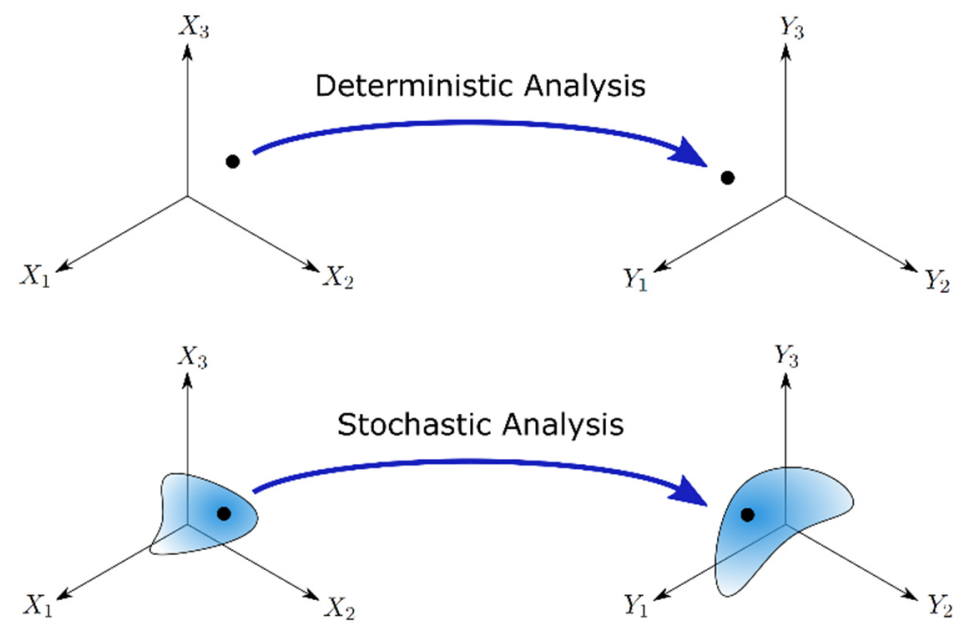

Bild 1: Deterministische Simulation vs. stochastische Simulation, nach [4]

Ein rein deterministisches Modell und deterministische Analyse erzeugen lediglich ein approximatives Ergebnis, denn in der Regel sind in jedem physikalischen System Parameter enthalten, deren Wert nicht exakt bekannt ist. Bereits geringe Variationen der Werte einzelner Parameter können eine große Auswirkung auf den Systemausgang bewirken. Diese Auswirkungen können bei der Anwendung deterministischer Simulationsmethoden nicht identifiziert werden. Mit Hilfe stochastischer Simulation können derartige Zusammenhänge jedoch erkannt werden. Die Ergebnisse sind daher präziser und aufschlussreicher, sodass letztendlich die richtigen Entscheidungen getroffen werden können.

In der vorliegenden Arbeit werden Unsicherheiten in der Anregung der Räder durch die Fahrt auf unebener Fahrbahn berücksichtigt. Hierbei handelt es sich um aleatorische Unsicherheit, welche mittels probabilistischer Methoden beschrieben werden kann. Die Wahrscheinlichkeitsverteilungen werden als bekannt angenommen. Die Unsicherheiten werden durch Zufallsvariablen beschrieben, mit dem Ziel, statistische Verteilungen der Systemausgänge zu erhalten. Beispiele für probabilistische Methoden sind, unter anderem, die Monte Carlo Methode, die Störungsmethode, und spektrale Methoden, wie KarhunenLoève Expansion und Polynomial Chaos Entwicklung. In dieser Arbeit wird Polynomial Chaos, insbesondere die Kollokationsmethode, als eine nicht sampling-basierte Methode angewandt, und die Ergebnisse mit der sampling-basierten Monte Carlo Methode hinsichtlich Genauigkeit und Rechenaufwand verglichen. 
Als Einführung in die stochastische Simulation soll das in Bild 2 gezeigte System mit einem Freiheitsgrad betrachtet werden.

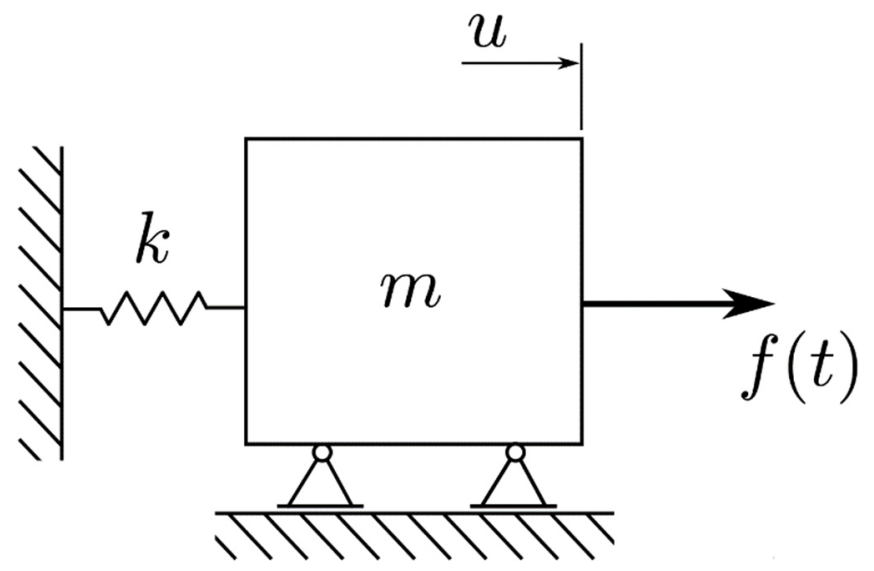

Bild 2: System mit einem Freiheitsgrad

Eine Masse $m$ ist mit einer Feder mit Federsteifigkeit $k$ verbunden. Es erfolgt eine Anregung durch die Kraft $f(t)$. Sofern das System deterministisch modelliert wird, ergibt sich die folgende Bewegungsgleichung:

$$
m \ddot{u}(t)+k u(t)=f(t)
$$

Die Systemantwort kann mit Hilfe analytischer oder numerischer Ansätze zur Lösung der Differentialgleichung gewonnen werden.

Ist hingegen eine Unsicherheit in der Steifigkeit $k$ der Feder bekannt und soll berücksichtigt werden, so ergibt sich

$$
m \ddot{u}(t, \xi)+k(\xi) u(t, \xi)=f(t)
$$

mit der Zufallsvariable $\xi$. Die Unsicherheit der Federsteifigkeit ist damit berücksichtigt, und eine bestimmte Verteilungsfunktion von $k(\xi)$ wird angenommen. Selbstverständlich können Unsicherheiten in mehreren Parametern, auch mit jeweils voneinander unabhängigen Wahrscheinlichkeitsverteilungen, berücksichtigt werden, sodass ein mehrdimensionaler Wahrscheinlichkeitsraum entsteht: $\xi=\left\{\xi_{1}, \xi_{2}, \ldots\right\}$.

\section{Die Monte Carlo Simulationsmethode}

Bei Anwendung der Monte Carlo Methode werden Stichproben des unsicheren Parameters gezogen. Im vorliegenden Beispiel ist dies die Steifigkeit $k$. Für jede gezogene Stichprobe wird ein Simulationslauf durchgeführt, um jeweils die zugehörige Systemantwort zu erhalten. Bei ausreichend großer Anzahl an Stichproben kann die Verteilung der Systemantwort sowie die 
statistischen Eigenschaften, wie zum Beispiel Erwartungswert und Varianz, gewonnen werden. Die Grafik in Bild 3 zeigt die Vorgehensweise für $N$ Stichproben.

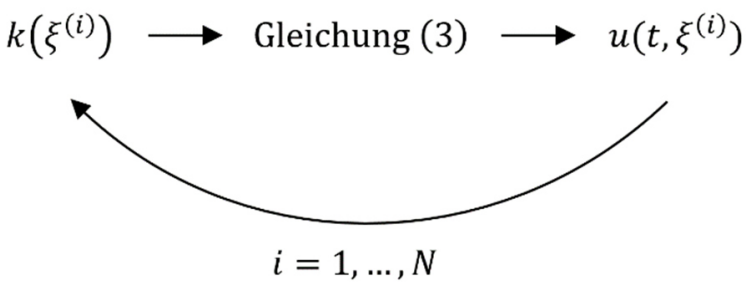

Bild 3: Prinzip der Monte Carlo Methode

Um aussagekräftige Simulationsergebnisse zu generieren, ist in der Regel eine sehr große Anzahl an Stichproben nötig. Für jede Stichprobe muss die Systemantwort mit dem originalen Simulationsmodell berechnet werden. Bei einer hohen Anzahl an Stichproben, welche durchaus im vier- oder fünfstelligen Bereich liegen kann, ergibt sich ein extrem hoher Rechenaufwand. Insbesondere bei der Verwendung detaillierter Simulationsmodelle mit zahlreichen Freiheitsgraden, bei welchen ein einziger Simulationslauf bereits eine hohe Rechenzeit in Anspruch nimmt, führt die Monte Carlo Methode zu nicht akzeptabel großem Rechenaufwand.

\section{Polynomial Chaos Theorie und die Kollokationsmethode}

Dieser Abschnitt soll als kurze Einführung in die Polynomial Chaos Entwicklung dienen. Ausführliche Informationen und Hintergründe können beispielsweise den Werken von Ghanem \& Spanos [5], Xiu \& Karniadakis [6] oder Sepahvand et al. [7] entnommen werden.

Die Unsicherheiten im System Ein- und Ausgang können mittels der Polynomial Chaos Entwicklung approximiert werden. Diese entspricht einer Entwicklung von orthogonalen Polynomen bezüglich der Zufallsvariablen. Der große Nutzen der Methode entsteht dadurch, dass die stochastische Systemgleichung (oder Gleichungssystem) durch ein System von deterministischen Gleichungen ersetzt wird, dessen Lösung eine Approximation der Lösung der stochastischen Systemgleichung (oder Gleichungen) darstellt. Während die Monte Carlo Methode aufwendiges Sampling erfordert, muss hier lediglich ein System aus deterministischen Gleichungen gelöst werden.

Die grundlegenden Ideen von Galerkin Projektion und Kollokationsmethode sollen im Folgenden kurz vorgestellt werden. Es soll ein komplettes Set orthogonaler Polynome $\psi$ betrachtet werden, welche Funktionen einer Zufallsvariable $\xi$ sind. $\xi=\left\{\xi_{1}, \xi_{2}, \ldots\right\}$ ist ein Vektor aus Zufallsvariablen $\xi_{i}$ mit jeweils zugehörigem Zufallsraum $\Omega_{i}$. Der Wahrscheinlichkeitsraum 
$(\Omega, F, P)$ mit dem Zufallsraum $\Omega$, der $\sigma$-Albebra $F$ bezüglich $\Omega$, und dem Wahrscheinlichkeitsmaß $P$ bezüglich $\Omega$ ist gegeben. Ein unsichererer Parameter $X: \Omega \rightarrow R$ mit endlicher Varianz kann demnach wie folgt ausgedrückt werden [6, 7]

$$
X=x_{0} \psi_{0}+\sum_{i_{1}=1}^{\infty} x_{i_{1}} \psi_{1}\left(\xi_{i_{1}}\right)+\sum_{i_{1}=1}^{\infty} \sum_{i_{2}=1}^{i_{1}} x_{i_{1} i_{2}} \psi_{2}\left(\xi_{i_{1}}, \xi_{i_{2}}\right)+\sum_{i_{1}=1}^{\infty} \sum_{i_{2}=1}^{i_{1}} \sum_{i_{3}=1}^{i_{2}} x_{i_{1} i_{2} i_{3}} \psi_{3}\left(\xi_{i_{1}}, \xi_{i_{2}}, \xi_{i_{3}}\right)+\cdots
$$

oder in der kompakten Form

$$
X=\sum_{i=0}^{\infty} x_{i} \psi_{i}(\xi)
$$

$x_{i}$ sind unbekannte deterministische Koeffizienten, auf deren Berechnung im Folgenden eingegangen wird. Multiplikation beider Seiten von Gleichung (4) mit einer Testfunktion $\psi_{j}(\xi)$ ergibt

$$
\begin{gathered}
\left\langle x(\xi), \psi_{j}(\xi)\right\rangle=\left\langle\sum_{i=0}^{\infty} x_{i} \psi_{i}(\xi), \psi_{j}(\xi)\right\rangle \\
\text { für } i=j: \quad x_{i}=\frac{\left\langle x(\xi), \psi_{i}(\xi)\right\rangle}{\left\langle\psi_{i}^{2}(\xi)\right\rangle} \quad i=0,1,2, \ldots
\end{gathered}
$$

Bei einem multidimensionalen Wahrscheinlichkeitsraum resultiert [7]

$$
x_{i}=\frac{1}{\left\langle\psi_{i}^{2}\right\rangle} \int_{\Omega_{1}} \int_{\Omega_{2}} \ldots \int_{\Omega_{n}} X \psi_{k}(\xi) \rho_{1}\left(\xi_{1}\right) \rho_{2}\left(\xi_{2}\right) \ldots \rho_{n}\left(\xi_{n}\right) \mathrm{d} \xi_{1} \mathrm{~d} \xi_{2} \ldots \mathrm{d} \xi_{n}
$$

wobei $\rho_{i}\left(\xi_{i}\right)$ die Wahrscheinlichkeitsdichtefunktion der Zufallsvariablen $\xi_{i}$ ist. Dieser Ansatz zur Bestimmung der deterministischen Koeffizienten wird auch intrusive Methode oder Galerkin Projektion genannt. Sobald die Koeffizienten bekannt sind, ist die Lösung des Problems abgeschlossen. Die Verteilung sowie die stochastischen Momente gehen direkt aus den Koeffizienten hervor. In der Praxis wird die unendliche Reihe in Gleichung (4) auf eine endliche Anzahl an Termen beschränkt:

$$
X \approx \sum_{i=0}^{N} x_{i} \psi_{i}(\xi)
$$

Demnach ist die Anzahl an Termen $N+1$, welche wie folgt berechnet werden kann [6]

$$
N+1=\frac{(n+p) !}{n ! p !}
$$

mit der Dimension $n$ der Zufallsvariable $\xi$. $p$ ist die höchste Ordnung der Polynome $\psi_{i}(\xi)$.

Bestimmte Typen von orthogonalen Polynomen haben Wichtungsfunktionen, die identisch sind mit den Wahrscheinlichkeitsfunktionen bestimmter Zufallsverteilungen. In der Praxis wird der Typ der Polynome deshalb unter Berücksichtigung der gegebenen Verteilung der 
Zufallsvariablen gewählt. Tabelle 1 zeigt die verschiedenen Typen orthogonaler Polynome zu den entsprechenden Zufallsvariablen.

Tabelle 1: Typ der Polynome und die entsprechenden Zufallsvariablen, nach [6]

\begin{tabular}{|l|l|c|}
\hline Zufallsvariable $\boldsymbol{\xi}$ & Typ der Polynome & Domain \\
\hline Gaussian & Hermite & $(-\infty, \infty)$ \\
\hline Gamma & Laguerre & {$[0, \infty)$} \\
\hline Beta & Jacobi & {$[a, b]$} \\
\hline Uniform & Legendre & {$[a, b$} \\
\hline
\end{tabular}

Neben der Galerkin Projektion kann zur Bestimmung der unbekannten, deterministischen Koeffizienten die nicht-intrusive Methode, auch Kollokationsmethode genannt, angewendet werden. Bei der Kollokationsmethode wird das Modell beziehungsweise die Systemgleichungen als „Black Box“ behandelt. Im Gegensatz zur Galerkin Projektion können daher auch nichtlineare Systeme sowie Systeme mit unbekannten Strukturdaten betrachtet werden.

Während der Fehler durch die Approximation bei der Galerkin Projektion im gesamten Domain minimiert wird, gilt dies bei der Kollokationsmethode nur an bestimmten Punkten, den sogenannten Kollokationspunkten $\xi^{(p)}[7]$

$$
\varepsilon\left(\xi^{(p)}\right)=0
$$

Die Kollokationspunkte werden bestimmt als die Nullstellen des Polynoms, welches eine Ordnung über dem höchsten für die Approximation verwendeten Polynoms ist. Als Beispiel soll Polynomial Chaos vierter Ordnung mit zwei unischeren Parametern $\xi_{1}$ und $\xi_{2}$ betrachtet werden. Es ergeben sich mit der höchsten Ordnung der Polynome $N+1=5$ fünf Nullstellen und demnach insgesamt $(N+1)^{p}=5^{2}=25$ Kollokationspunkte, da die Nullstellen für die zwei Dimensionen für alle Möglichkeiten kombiniert werden.

Anschließend wird mit dem originalen Simulationsmodell ein Lauf für jeden Kollokationspunkt $\xi^{(p)}$ durchgeführt.

$$
\begin{gathered}
\xi^{(1)} \rightarrow \text { Modell } \rightarrow F^{(1)} \\
\vdots \\
\xi^{(25)} \rightarrow \text { Modell } \rightarrow F^{(25)}
\end{gathered}
$$

Als Ergebnis ergibt sich ein Vektor $F(\xi)$, oder bei Zeit- oder Ortsabhängigkeiten (*) eine Matrix $F(*, \xi)$. Der Polynomial Chaos Ansatz 


$$
F(*, \xi)=\sum_{j=0}^{N} f_{j}(*) \psi_{j}(\xi)
$$

kann als Gleichungssystem in Matrix-Form dargestellt werden

$$
\begin{gathered}
\left\{\begin{array}{c}
F^{(1)} \\
\vdots \\
F^{(25)}
\end{array}\right\}=[A]\left\{\begin{array}{c}
f_{0} \\
f_{1} \\
\vdots \\
f_{15}
\end{array}\right\} \\
{[A]=\left[\psi\left(\xi^{(i)}\right)\right]_{25 \times 15}, \quad i=1,2, \ldots, 25}
\end{gathered}
$$

Dieses System deterministischer Gleichungen kann sehr effizient gelöst und somit die unbekannten Koeffizienten $f_{j}$ berechnet werden. Mit der Bestimmung der Koeffizienten ist das Problem vollständig gelöst, da die statistischen Eigenschaften des Systemausgangs $F$ unmittelbar aus den Koeffizienten $f_{j}$ bestimmt werden können.

\section{Mehrkörpermodell / Modellbildung des PKW}

Zur Untersuchung des Schwingungsbelastung der Insassen eines PKW bei Fahrt über unebene Fahrbahn wird das in Bild 4 dargestellte Mehrkörpersimulationsmodell mit acht Freiheitsgraden verwendet.

Das Modell besteht aus der Karosserie, je einer Radaufhängung pro Rad, vier Reifen und dem Fahrersitz. Die ungefederten Massen $m_{1 F}$ und $m_{1 R}$ sowie der Passagier $m_{p}$ besitzen einen Freiheitsgrad, da nur eine Bewegung in vertikaler Richtung zugelassen ist. Die Karosserie $m_{B}$ kann sich neben einer Bewegung in vertikaler Richtung zusätzlich in die Richtungen $\phi$ und $\theta$ verdrehen. Somit ergeben sich insgesamt acht Freiheitsgrade.

An dieser Stelle soll angemerkt werden, dass es sich bei dem vorliegenden Modell um eine stark vereinfachte Abbildung eines PKW handelt. Dies liegt unter anderem daran, dass die meisten Körper des Modells in der Realität aus zahlreichen Komponenten bestehen, wie beispielsweise die ungefederten Massen. Des Weiteren sind sämtliche Nichtlinearitäten in Federn und Dämpfern nicht berücksichtigt. Zudem führt die Vernachlässigung der Flexibilität einzelner Körper zu einer begrenzten Aussagefähigkeit der Ergebnisse, die mit dem 8-Freiheitsgrad-Modell generiert werden. Insbesondere die Flexibilität der Karosserie mit ihren Eigenfrequenzen und Eigenschwingformen hat großen Einfluss auf die Übertragung von Vibrationen von den Rädern zum Fahrersitz.

Das stark vereinfachte Simulationsmodell stellt jedoch kein Problem dar. Das zentrale Thema 


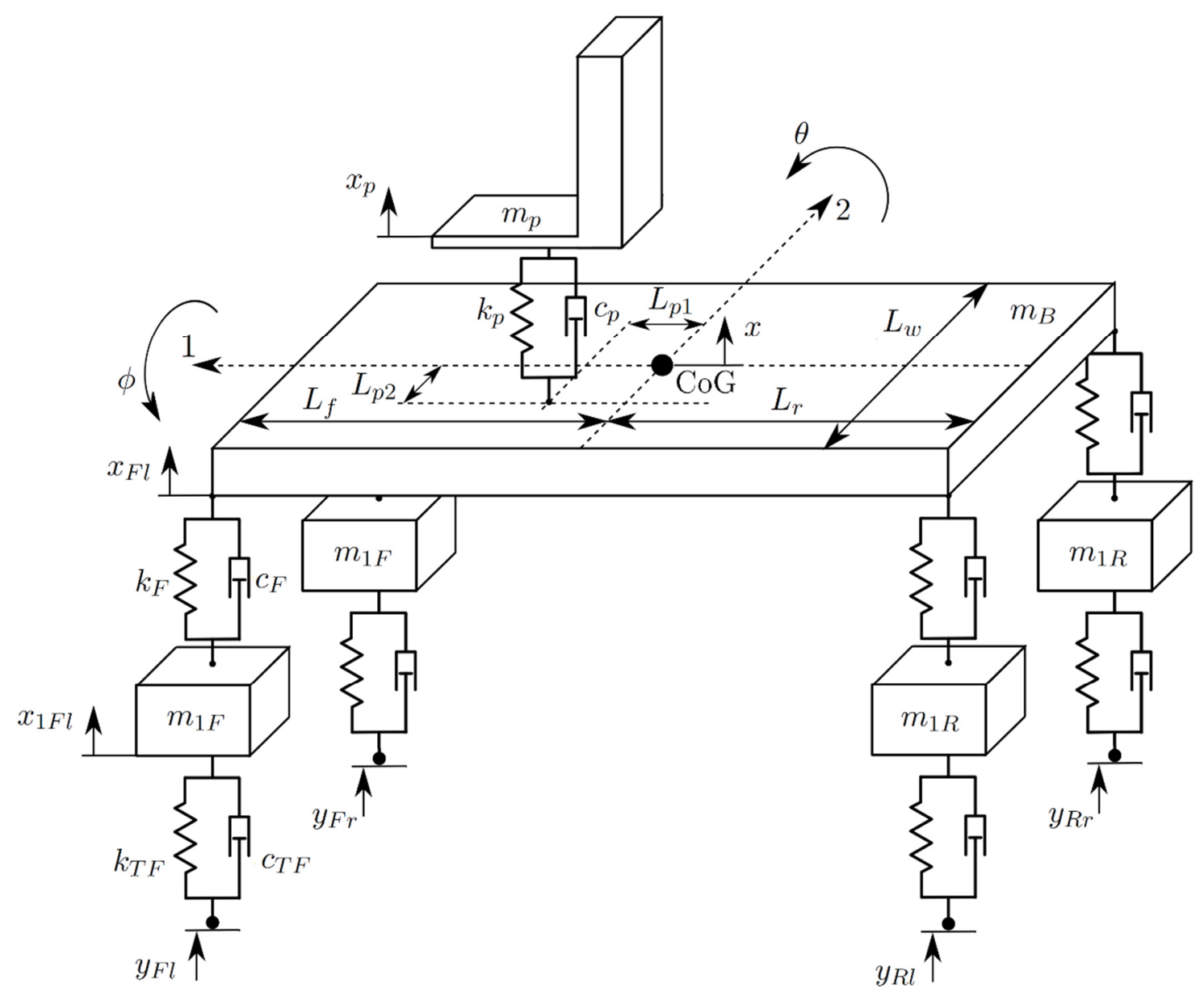

Bild 4: Mehrkörpersimulationsmodell des PKW mit acht Freiheitsgraden, nach [8]

dieser Arbeit ist die Bewertung der Vibrationsbelastung in einem PKW durch die Anwendung einer nicht sampling-basierten Simulationsmethode zur Berücksichtigung von Unsicherheiten in der Schwingungsanregung an den Rädern. Die vorgestellte Simulationsmethode ist unabhängig vom Detaillierungsgrad des Modells. Wie in Abschnitt 2 erläutert, behandelt die Kollokationsmethode das originale Simulationsmodell als „Black Box“. Daher kann der hier angewandte Ansatz genauso für detaillierte Mehrkörpersimulationsmodelle mit einer sehr großen Anzahl an Freiheitsgraden, wie sie beispielsweise von Automobilherstellern verwendet werden, genutzt werden.

\section{Simulation und Ergebnisse}

Unter Verwendung des im vorherigen Abschnitt vorgestellten Mehrkörpermodells kann die Schwingungsbelastung der Insassen eines PKW bei Fahrt über unebenen Untergrund ermittelt werden. Nach Untersuchung verschiedener Szenarien sollen hier exemplarisch die 
Ergebnisse für eine Fahrt über eine wellige Landstraße gezeigt und diskutiert werden. Die Anregung an den Rädern ist als reine Weganregung modelliert und durch eine Sinusfunktion approximiert. Für eine einfache Interpretation werden hier Ergebnisse gezeigt, die aus Untersuchungen mit lediglich einem unsicheren Parameter, der Amplitude $A$, resultieren.

$$
y(t, \xi)=A(\xi) \sin (\omega t)
$$

Zusätzlich zur Amplitude kann beispielsweise auch die Anregungsfrequenz $\omega$ als unsicherer Parameter behandelt werden, um noch aussagekräftigere Ergebnisse zu generieren.

Die zufällige Amplitude wird im vorliegenden Fall als normalverteilt angenommen. Dieser unsichere Eingangsparameter kann daher mit Polynomial Chaos Entwicklung auf Basis von Hermite Polynomen approximiert werden.

Der Erwartungswert $\mu$ der Amplitude wird auf $10 \mathrm{~mm}$ festgelegt. Die Standardabweichung $\sigma$ soll $1 \mathrm{~mm}$ betragen, was $10 \%$ des Erwartungswertes entspricht. Für die Geschwindigkeit des Autos wird $70 \mathrm{~km} / \mathrm{h}$ angenommen. Bei einer Wellenlänge der Straße von ca. $10.2 \mathrm{~m}$ ergibt sich eine Anregungsfrequenz $\omega$ von $12 \mathrm{rad} / \mathrm{s}$.

$\mathrm{Da}$ die Schwingungsbelastung des Passagiers bewertet werden soll, muss zunächst die Beschleunigung des Sitzes in vertikaler Richtung bestimmt werden. In Bild 5 und Bild 6 sind die gesuchten Beschleunigungen am Sitz in vertikaler Richtung über der Zeit sowie über der Frequenz dargestellt. Die Ergebnisse im Frequenzbereich resultieren aus einer Fast Fourier Transformation der Ergebnisse aus den Simulationsläufen des originalen Modells und anschließender Anwendung der Kollikationsmethode. Die zugehörigen Polynomial Chaos Koeffizienten sind daher in diesem Fall nicht zeit-, sondern frequenzabhängig (siehe auch Bild 8).

Die beiden Plots in Bild 5 und Bild 6 zeigen die mittels stochastischer Simulation generierten Ergebnisse für die Beschleunigung des Sitzes in vertikaler Richtung. Zum Vergleich ist sowohl das Ergebnis aus der Kollokationsmethode, als auch die mittels sampling-basierter Monte Carlo Methode gewonnene Systemantwort gezeigt.

Der schwarze Graph stellt jeweils die deterministische Lösung dar. Die blaue und rote Fläche umfasst den Bereich aller Lösungen, die für beide Simulationsmethoden aufgrund der Unsicherheit in der Anregung entstehen. Sowohl im Zeitbereich, als auch im Frequenzbereich, überdecken sich die Bereiche der Lösungen aus Kollokationsmethode und Monte Carlo Methode fast perfekt. Der Kollokationsmethode kann daher bereits an dieser Stelle eine hohe Genauigkeit attestiert werden. Während mit dem originalen Simulationsmodell bei Anwendung der Monte Carlo Methode 1000 Simulationsläufe nötig sind, sind bei der Kollokationsmethode im vorliegenden Fall nur fünf Läufe für fünf Kollokationspunkte erforderlich. Anschließend kann 


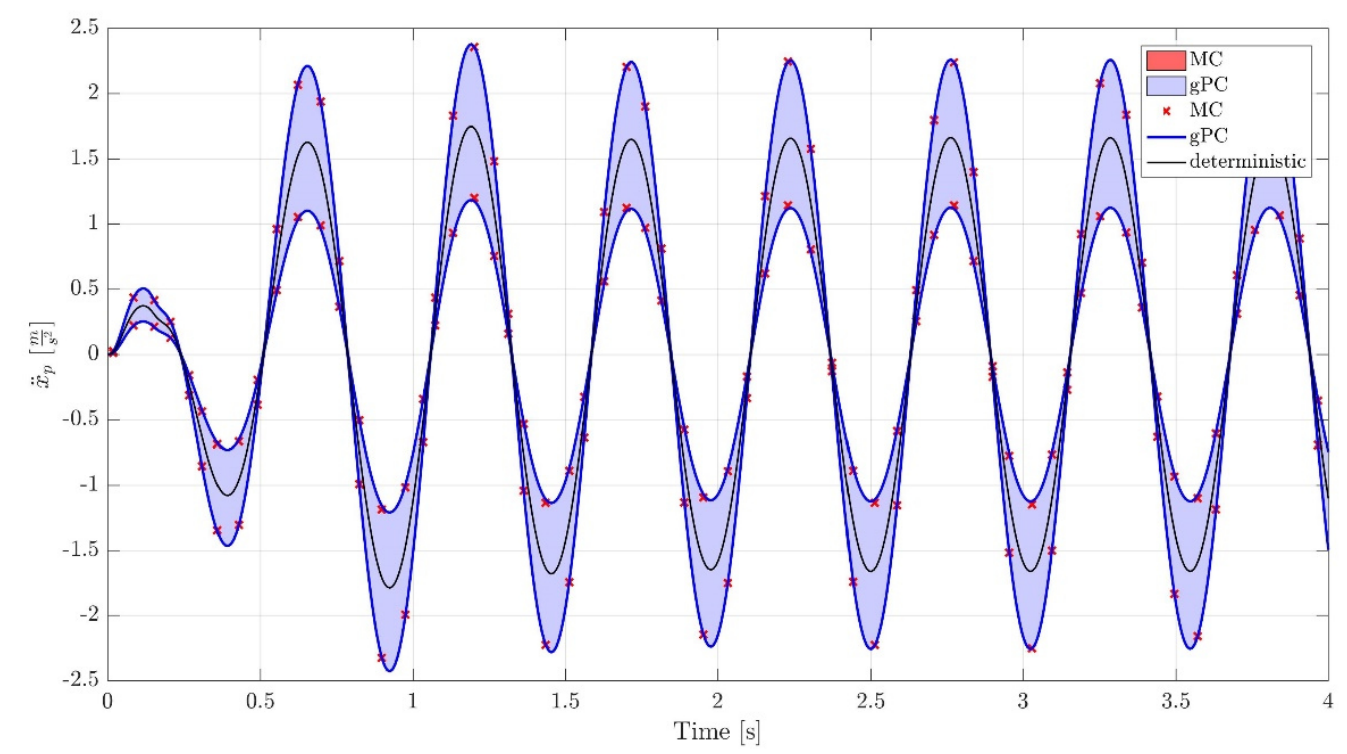

Bild 5: Beschleunigungen am Fahrersitz über der Zeit. Kollokationsmethode (dritte Ordnung, 5 Kollokationspunkte, 1000 Realisierungen) vs. Monte Carlo Methode (1000 Samples)

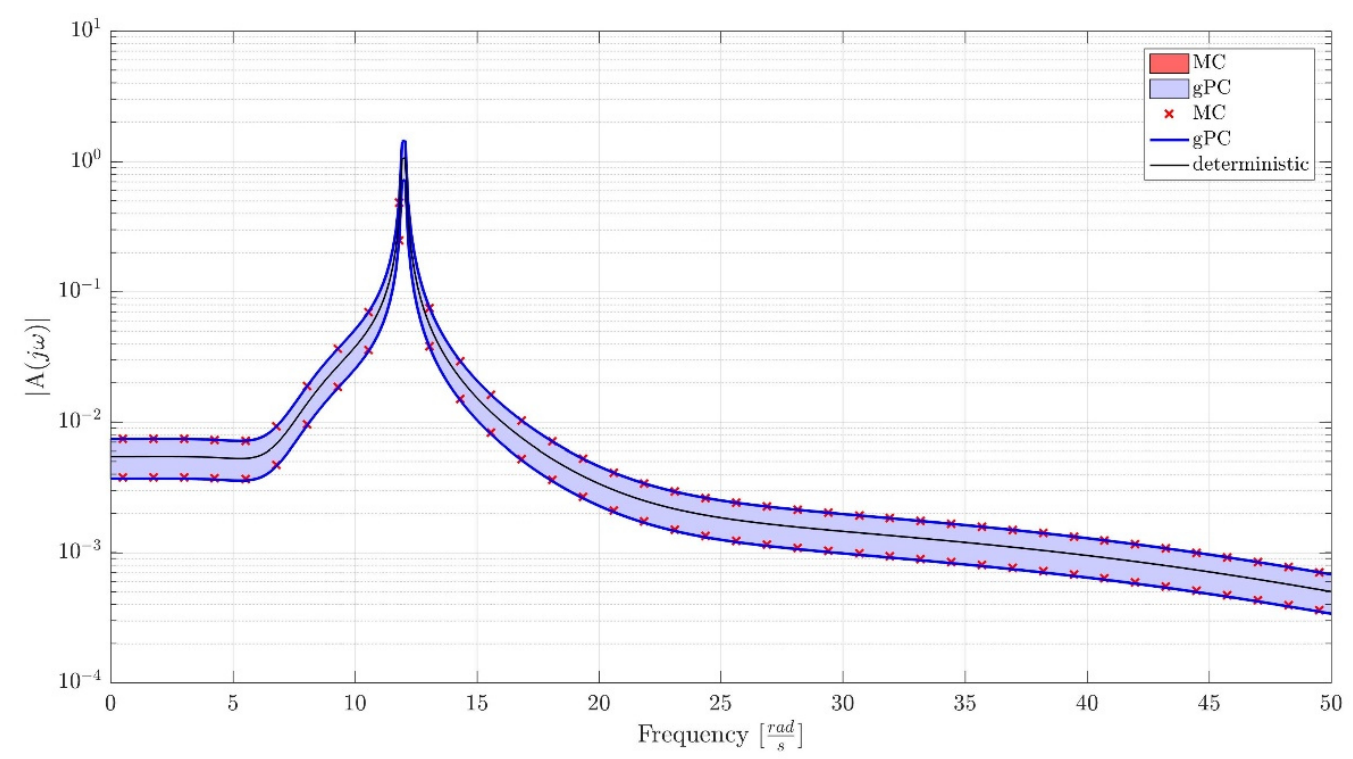

Bild 6: Beschleunigungen am Fahrersitz über der Frequenz. Kollokationsmethode (dritte Ordnung, 5 Kollokationspunkte, 1000 Realisierungen) vs. Monte Carlo Methode (1000 Samples)

sehr effizient eine große Anzahl an Realisierungen (hier 1000) mit dem approximierten Modell durchgeführt werden. Zwischen den Rechenzeiten der nicht sampling-basierten Kollokationsmethode und der Monte Carlo Methode liegen im vorliegenden Fall Faktoren um 30. Bei Anwendung größerer, detaillierterer Simulationsmodelle können je nach Anzahl unsicherer 
Parameter noch wesentlich größere Faktoren resultieren und somit noch signifikantere Einsparungen hinsichtlich des Rechenaufwands erreicht werden.

Die Plots in Bild 7 und Bild 8 zeigen, dass die gewählte dritte Ordnung für die Polynomial Chaos Entwicklung für diese Problemstellung ausreicht. Lediglich die ersten beiden Koeffizienten nehmen signifikante Werte an, höhere Koeffizienten gehen gegen Null. Dies kann durch den vorliegenden „optimalen Fall“ begründet werden, da hier die Wichtungsfunktion der orthogonalen Polynome der Dichtefunktion des unsicheren Parameters entspricht.

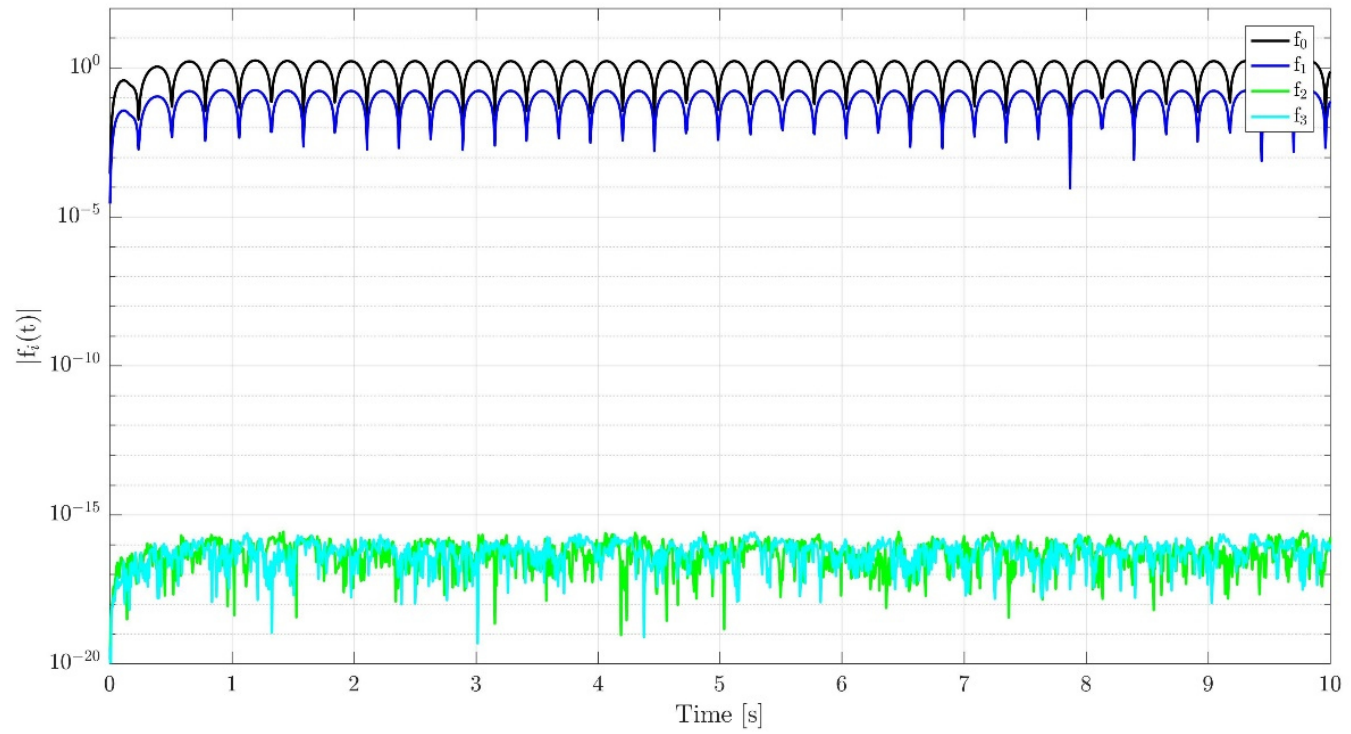

Bild 7: Koeffizienten von Polynomial Chaos dritter Ordnung über der Zeit

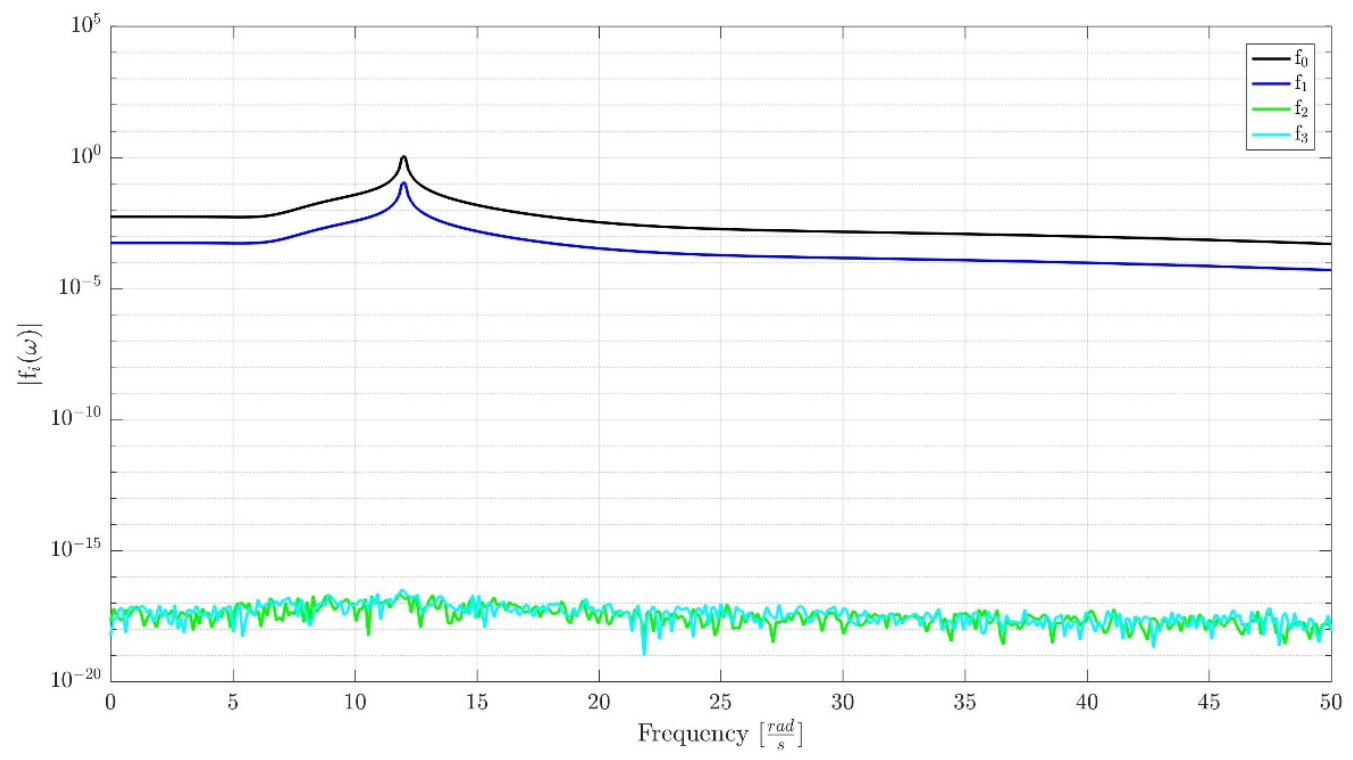

Bild 8: Koeffizienten von Polynomial Chaos dritter Ordnung über der Frequenz 
Mit den gewonnenen Ergebnissen kann schließlich der Schwingungsdosiswert VDV bestimmt werden. Der Frequenzwichtungsfaktor wird gemäß ISO 2631-1 [1] zu 0.531 gewählt. Das Ergebnis für eine 30-minütige Fahrt des PKW über die wellige Landstraße ist in Bild 9 gezeigt.

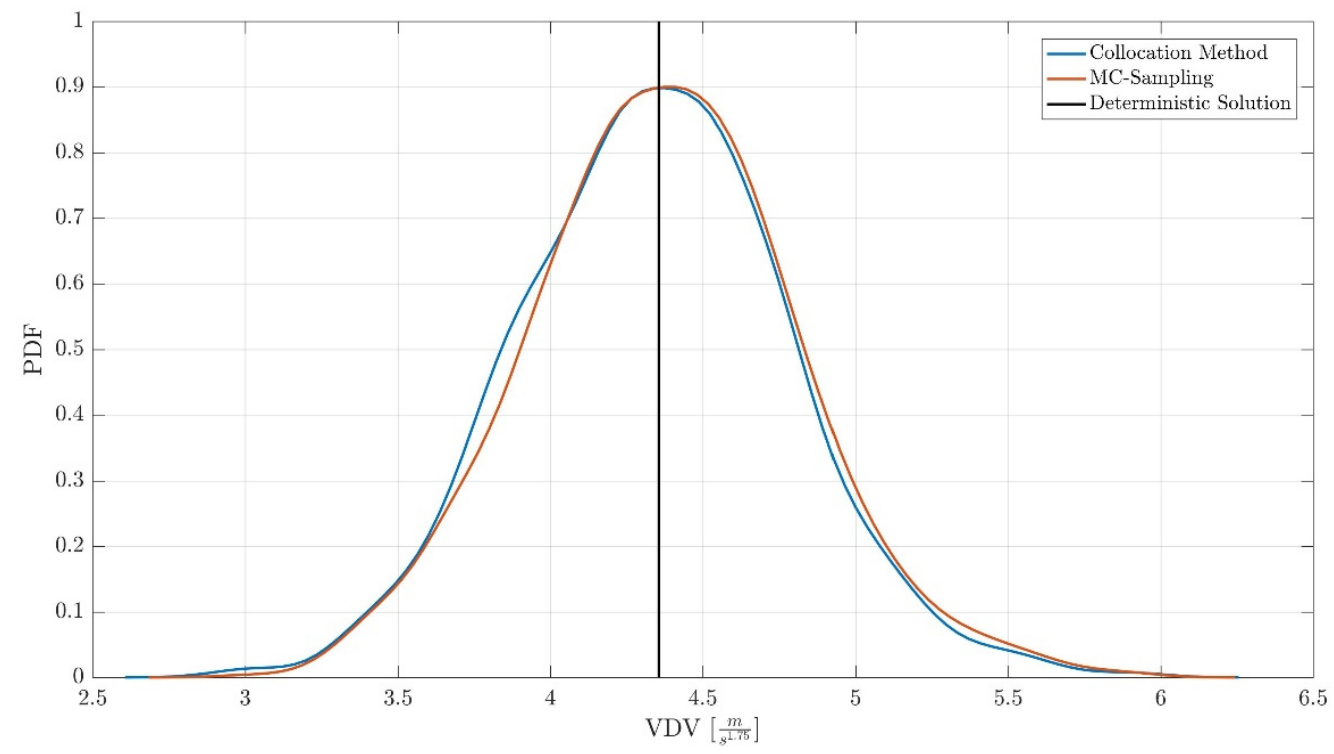

Bild 9: Dichtefunktion des Schwingungsdosiswertes für eine 30-minütige Fahrt. Kollokationsmethode (dritte Ordnung, 5 Kollokationspunkte, 1000 Realisierungen) vs. Monte Carlo Methode (1000 Samples)

Auch in diesem Plot ist die gute Genauigkeit der Kollokationsmethode klar erkennbar. Die Graphen aus Kollokationsmethode und Monte Carlo Methode unterscheiden sich nur minimal. Des Weiteren wird die Mächtigkeit stochastischer Simulation deutlich. Aufgrund der Unsicherheiten in der Anregung resultiert eine deutliche Streuung im Systemausgang. Mittels deterministischer Simulation kann lediglich ein Nominalwert von ca. $4.3 \mathrm{~m} / \mathrm{s}^{1.75}$ für den Schwingungsdosiswert gefunden werden, während durch die Anwendung stochastischer Simulation die Unsicherheit im Systemausgang aufgrund der Unsicherheit im Systemeingang identifiziert werden kann. Somit ist bei Anwendung stochastischer Simulation eine weitaus bessere Bewertung der Schwingungsbelastung der Insassen eines PKW möglich.

\section{Zusammenfassung}

Die gezeigten Ergebnisse unterstreichen die Wichtigkeit der Anwendung stochastischer Simulation für Problemstellungen mit Parameterunsicherheiten. Es ist offensichtlich, dass bei der Bewertung der Schwingungsbelastung der Insassen eines PKW verschiedene Parameter Unsicherheiten enthalten. Die Anwendung sampling-basierter Methoden ist unmittelbar mit 
einem sehr hohen Rechenaufwand verbunden. Insbesondere beim Einsatz detaillierter Simulationsmodelle mit einer großen Zahl an Freiheitsgraden ergeben sich dadurch inakzeptabel hohe Rechenzeiten. Die Ergebnisse dieser Arbeit bestätigen jedoch die Anwendbarkeit und die Genauigkeit nicht sampling-basierter Simulationsmethoden, welche verhältnismäßig kleine Rechenzeiten in Anspruch nehmen. Die Anwendung der Kollokationsmethode wird daher empfohlen, und es wird angenommen, dass die Verbreitung der Anwendung nicht sampling-basierter Methoden für stochastische Simulation in der Industrie nur eine Frage der Zeit darstellt.

Besonders für die Bewertung der Schwingungsbelastung bei der Fahrt in einem PKW ist eine umfassende Analyse und Berechnung unumgänglich, insbesondere in Zeiten, in denen die Perfektion des Fahrkomforts im PKW immer weiter in den Mittelpunkt rückt.

[1] International Organization for Standardization. ISO 2631-1. Mechanical vibration and shock - evaluation of human exposure to whole-body vibration - part 1: general requirements. ISO, 1997.

[2] A. Batou, C. Soize, C. K. Choi, und H. H. Yoo. Robust design in multibody dynamics application to vehicle ride-comfort optimization. Procedia lutam, 13:90-97, 2015.

[3] J. Vom Scheidt, R. Wunderlich, und B. Fellenberg. Random road surfaces and vehicle vibration. Progress in industrial mathematics at ECMI, 98:352-359, 1999.

[4] E. Patelli, M. Broggi, M. de Angelis, und M. Beer. Opencossan: An efficient open tool for dealing with epistemic and aleatory uncertainties. In Vulnerability, Uncertainty, and Risk: Quantification, Mitigation, and Management, Seiten 2564-2573. ASCE, 2014.

[5] R. G. Ghanem und P. D. Spanos. Stochastic Finite Elements: A Spectral Approach. Springer-Verlag, New York, 1991.

[6] D. Xiu und G. E. Karniadakis. The wiener-askey polynomial chaos for stochastic differential equations. SIAM journal on scientific computing, 24(2):619-644, 2002.

[7] K. Sepahvand, S. Marburg, und H. J. Hardtke. Uncertainty quantification in stochastic systems using polynomial chaos expansion. International Journal of Applied Mechanics, 2(02):305-353, 2010.

[8] A. Shirahatt, P. Prasad, P. Panzade, und M. M. Kulkarni. Optimal design of passenger car suspension for ride and road holding. Journal of The Brazilian Society of Mechanical Sciences and Engineering - J BRAZ SOC MECH SCI ENG, 30, 032008. 